\title{
New result on phase shift analysis
}

\author{
André Martin" \\ Physics Department, CERN, CH-1211 Geneve 23, Switzerland \\ Jean-Marc Richard $\odot^{\dagger}$ \\ Université de Lyon, Institut de Physique des 2 Infinis de Lyon UCBL-IN2P3-CNRS, \\ 4, rue Enrico Fermi, F-69622 Villeurbanne, France
}

(Received 23 April 2020; accepted 1 May 2020; published 14 May 2020)

\begin{abstract}
Assuming a certain continuity property, we prove, using the old results of Itzykson and Martin, that, except for an obvious ambiguity, there are only at most two amplitudes reproducing an elastic differential cross section at a given energy.
\end{abstract}

DOI: 10.1103/PhysRevD.101.094014

\section{HISTORICAL INTRODUCTION}

Given a differential cross section at one energy in the elastic region, can one find the scattering amplitudes? We restrict ourselves to the spinless case. Forgetting kinematical factors (more precisely, we neglect $1 / k$ in the amplitude and $4 \pi / k^{2}$ in the integrated cross section), the scattering amplitude is given by

$$
f(\cos \theta)=\sum_{\ell}(2 \ell+1) f_{\ell} P_{\ell}(\cos \theta),
$$

with $f_{\ell}=\sin \delta_{\ell} \exp \left(i \delta_{\ell}\right)$. The $\delta_{\ell}$ 's are the phase shifts. The differential cross section is given by $F^{2}$, where

$$
F=|f(\cos \theta)|
$$

It seems that it was first realized by T Y. Wu and T. Ohmura in 1962 [1] that the phase $\phi$ of the scattering amplitude such that

$$
f(\cos \theta)=F(\cos \theta) \exp [i \phi(\cos \theta)]
$$

satisfies a nonlinear integral equation which, using numbers to designate directions, is

$$
\begin{aligned}
& F(12) \sin \phi(12) \\
& \quad=\frac{1}{4 \pi} \int d \Omega_{3} F(13) F(23) \cos [\phi(13)-\phi(23)] .
\end{aligned}
$$

\footnotetext{
j-m.richard@ipnl.in2p3.fr

andre.martin@cern.ch
}

Published by the American Physical Society under the terms of the Creative Commons Attribution 4.0 International license. Further distribution of this work must maintain attribution to the author(s) and the published article's title, journal citation, and DOI.
However, they did not go very far in the possible solutions of this equation. One obvious ambiguity is that one can change the sign of all phase shifts or, equivalently change $f$ to $-f^{*}$, but the question is whether (1) has one or more solutions. J.H. Crichton, in 1966, pointed out that there are cases where the solution of (1) is not unique [2] and he exhibited a twofold ambiguity in the simple situation where there are only 3 partial waves, $\ell=0,1,2$. Later, in 1968, A. Martin [3] and R. Newton [4] found a condition under which the solution of (1) is unique and reached by a contraction mapping. This condition was:

$$
\sup \frac{F(13) F(23)}{F(12)}<0.79 \text {. }
$$

Later A. Gangal and J. Kupsch [5] succeeded in replacing 0.79 by 0.89 . Nobody realized that condition (5) implies $\sup F(11)<0.79$, and by the optical theorem,

$$
\sigma_{\text {tot }}<0.79 \text {. }
$$

What happens if this condition is violated? One can go a little further as was done by C. Itzykson and A. Martin [6] assuming that there is a finite number of partial waves and showing that if the cross section is less than 1.38, the solution is unique. Since the maximum $\ell$ is arbitrarily large, we believe that this holds also for an infinite number of partial waves. In fact one can go further than that and show that for a given total cross section $\sigma$, the maximum number of solutions is less than $2^{8 \sigma / 7}$ (see Appendix A). However, after the Crichton example, it was discovered that there are other situations with 2 and only 2 solutions. Courageously F. Berends and S. Ruijsenaars [7] and independently $\mathrm{H}$. Cornille [8] proved that if $\ell=0,1,2,3$, there are only 2 solutions, and H. Cornille and J.-M. Drouffe 
made the "tour de force" to prove the same for maximum $\ell=4$ [9].

C. Itzykson and A. Martin [6] undertook a different approach, assuming that the scattering amplitude is a genuine entire function, i.e., not a polynomial, and succeeded to prove that in most cases there are not more than 2 solutions. In this paper we shall utilize their result to go further. First we give a short lecture on entire functions in the next section, then we rederive some of the results of [6]. In the following section we modify a polynomial amplitude, to make it an entire function and succeed to prove that for polynomial amplitudes of arbitrarily large degree there are only 2 solutions. Finally, we move to amplitudes with infinite number of partial waves and succeed, we believe, in proving that there are only 2 solutions.

\section{LECTURE ON ENTIRE FUNCTIONS}

We begin by recalling some facts on entire functions which can be found in the book by Boas [10]. An entire function, $f(z)$ is analytic in the full complex plane. This, of course, includes polynomials. We are interested only in genuine entire functions which are such that if $M(r)$ is the maximum of $|f(z)|$ on $|z|=r$

$$
\frac{\ln M(r)}{\ln r} \rightarrow \infty \text { for } r \rightarrow \infty .
$$

An entire function is of order $\rho$ if $\lim \ln M(r) / r^{\rho}$ is finite. For instance $\exp z$ is of order 1 . A product of 2 functions of order $\rho$ is also of order $\rho$. An alternative definition is obtained from a power series expansion of $f$

$$
f=\sum_{n} a_{n} z^{n}
$$

Then

$$
\rho=\lim \sup _{n} \frac{n \ln n}{-\ln a_{n}} .
$$

An important property of functions of order strictly less than 1 is that they can be written as a convergent product over the zeros of the function

$$
f(z)=z^{m} \prod_{i}\left(1-z / z_{i}\right)
$$

This will be the case of functions of order $1 / 2$ like

$$
f=\cos (\sqrt{z})=\prod_{n}\left[1-\frac{z}{((n+1 / 2) \pi)^{2}}\right] .
$$

Let us point out that the only functions of order 1 which have no zero are proportional to $\exp (c z)$.
Now, we can also define entire functions as Legendre polynomial expansions.

$$
f(z)=\sum_{\ell}(2 \ell+1) f_{\ell} P_{\ell}(z) .
$$

The connection between polynomial expansion and power-series expansion is rather obvious because of the inequalities

$$
|z|^{\ell}<\left|P_{\ell}(z)\right|<(1+\sqrt{2})^{\ell}|z|^{\ell},
$$

for $\mid z]>1$ (see Appendix B). So, again, the order of the expansion (12) is given by

$$
\rho=\lim \sup _{\ell} \frac{\ell \ln (\ell)}{-\ln \left|f_{\ell}\right|} .
$$

\section{SOME RESULTS OF ITZYKSON-MARTIN [6]}

We consider only the situation where the scattering amplitude is an entire function of order 1 . It is given by (12). The unitarity condition is

$$
\operatorname{Im} f_{\ell}=\left|f_{\ell}\right|^{2} .
$$

If $f$ is entire, of order 1

$$
\lim \sup _{\ell} \frac{\ell \ln \ell}{-\ln \left|f_{\ell}\right|}=1 \text {. }
$$

Then

$$
\lim \sup _{\ell} \frac{\ell \ln \ell}{-\ln \left(\operatorname{Im} f_{\ell}\right)}=\frac{1}{2}
$$

So the absorptive part

$A=\sum_{\ell}(2 \ell+1) \operatorname{Im} f_{\ell} P_{\ell}(z)=\frac{1}{2 i}\left[f(z)-f^{*}\left(z^{*}\right)\right]$,

is of order $1 / 2$, while the dispersive part

$D=\sum_{\ell}(2 \ell+1) \operatorname{Re} f_{\ell} P_{\ell}(z)=\frac{1}{2}\left[f(z)+f^{*}\left(z^{*}\right)\right]$,

is of order 1.

Suppose we have 2 amplitudes with the same differential cross section:

$f=D+i A, \quad f^{\prime}=D^{\prime}+i A^{\prime}, \quad|f|^{2}=\left|f^{\prime}\right|^{2}$,

then 


$$
D^{2}-D^{\prime 2}=A^{\prime 2}-A^{2}=Q .
$$

$Q$ is a function of order $1 / 2$ and, therefore, can be written as a convergent product

$$
Q=z^{p} \prod_{i}\left(1-z / z_{i}\right) .
$$

But

$$
-Q=\left(D^{\prime}-D\right)\left(D^{\prime}-D\right) .
$$

The zeros of $D^{\prime}-D$ and $D^{\prime}+D$ form two complementary subsets of the zeros of $Q$. So

$$
D^{\prime}-D=M(z) z^{m} \prod_{\alpha}\left(1-z / z_{\alpha}\right),
$$

where $M(z)$ has no zero and the product is still convergent and of order $1 / 2$. Similarly

$$
D^{\prime}+D=N(z) z^{n} \prod_{\beta}\left(1-z / z_{\beta}\right),
$$

where $N(z)$ has no zero. $M$ and $N$ being of order 1 without zeros can only be of the form $\exp (c z), \exp \left(c^{\prime} z\right)$, but since they disappear in the product, $c^{\prime}=-c$. So

$$
2 D=\exp (c z) m(z)+\exp (-c z) n(z),
$$

where $m(z)$ and $n(z)$ are of order $1 / 2$.

Suppose now that we have a third amplitude, $f "=D "+i A$ ". Then $D$ and $D$ " will have a different decomposition:

$$
\begin{aligned}
2 D & =\exp (d z) p(z)+\exp (-d z) q(z) \\
& =\exp (c z) m(z)+\exp (-c z) n(z) .
\end{aligned}
$$

Suppose $c>d>0$. Then the term containing $\exp (c z)$ dominates. The second equality is impossible and necessarily, $c=d$. Then we have

$$
\exp (2 c z)(m-p)+n-q=0,
$$

but $m, p, n$, and $q$ being of order $1 / 2$ this is impossible. So the decomposition (26) is unique, and there are at most two solutions.

\section{AMBIGUITIES FOR POLYNOMIAL AMPLITUDES}

We consider an amplitude

$$
\begin{aligned}
\mathcal{F}_{L}= & \sum_{\ell=0}^{L}(2 \ell+1) f_{\ell} P_{\ell}(\cos \theta)=D_{L}+i A_{L}, \\
& \text { with } \operatorname{Im} f_{\ell}=\left|f_{\ell}\right|^{2},
\end{aligned}
$$

where $L$ is arbitrary. We shall complete this amplitude by a unitary amplitude extending from $L+1$ to infinity and manufacture in this way an entire function of order 1 . We add to $\mathcal{F}_{L}$ :

$$
\begin{array}{r}
R_{L}(\lambda)=\sum_{L+1}^{\infty}(2 \ell+1) r_{\ell}(\lambda) P_{\ell}(\cos \theta), \\
\text { with } \quad r_{\ell}(\lambda)=\operatorname{Re} r_{\ell}+i \operatorname{Im} r_{\ell},
\end{array}
$$

and we take, with $|\lambda|<1 / 2$,

$$
\operatorname{Rer}_{\ell}=\frac{\lambda}{2} \int_{-1}^{+1} P_{\ell}(x) \exp x d x .
$$

Though this can be calculated explicitly term by term, we need only an asymptotic estimate for large $\ell$ (see Appendix B).

$$
\operatorname{Re} r_{\ell} \simeq \frac{1}{2} \frac{1}{\ell !} \frac{\lambda}{2^{\ell}} \frac{\sqrt{\pi}}{\sqrt{\ell-1 / 2}} .
$$

The dispersive part of the new amplitude is:

$$
D_{L}+\sum_{L+1}^{\infty}(2 \ell+1) \operatorname{Re}_{\ell} P_{\ell}(z) .
$$

It is an entire function of order 1 . The absorptive part is constructed to satisfy elastic unitarity for all $\ell$ 's. So, for $\ell \leq L+1$,

$$
\operatorname{Im} r_{\ell}(\lambda)=\frac{1-\sqrt{1-4\left(\operatorname{Re} r_{\ell}\right)^{2}}}{2} .
$$

Notice that as soon as

$$
\operatorname{Re} r_{\ell}<\frac{2}{5}
$$

we have

$$
\operatorname{Im} r_{\ell}<\frac{5}{4}\left|\operatorname{Re} r_{\ell}\right|^{2} .
$$

So, asymptotically

$$
\operatorname{Im} r_{\ell}<\frac{5}{4}\left(\frac{1}{2} \frac{1}{\ell !} \frac{1}{2 \ell}\right)^{2} \frac{\lambda^{2} \pi}{\ell-1 / 2} .
$$

This means that the new absorptive part of the amplitude

$$
A_{L}+\sum_{L+1}^{\infty}(2 \ell+1) \operatorname{Im} r_{\ell} P_{\ell}(z),
$$

is an entire function of order $1 / 2$. So the Itzykson-Martin theorem applies to this amplitude, and there cannot be more 
than one amplitude giving the same differential cross section. The differential cross section is a continuous function of $\lambda$, including $\lambda=0$. it is even real analytic in a neighborhood of $\lambda=0$. We do not see how extra amplitudes (in finite number from Appendix A) could appear at $\lambda=0$. If they did, they should also be present for $\lambda$ different from zero. This is a continuity assumption, which seems to us reasonable, but which could be criticized.

\section{AMBIGUITIES FOR NONPOLYNOMIAL AMPLITUDES}

Suppose we have an amplitude which is neither a polynomial nor an entire function. This amplitude could exist on the interval $[-1,+1]$ or be analytic in the complex plane with some singularities: Its partial wave expansion will converge in an ellipse with foci $\{-1,+1\}$ touching the nearest singularity. This is the case in local field theory [11]. Then with $f_{\ell}=\sin \delta_{\ell} \exp \left(i \delta_{\ell}\right)$,

$\operatorname{Re} f_{\ell}<\exp (-k \ell), \quad$ and $\operatorname{Im} f_{\ell}<\exp (-2 k \ell)$.

Now we replace $\delta_{\ell}$ by $\delta_{\ell} \exp (-\lambda \ell \ln \ell)$ and correspondingly $f_{\ell}$ by $f_{\ell}(\lambda)$. The new dispersive part $D(\lambda)$ is an entire function of order 1 and the absorptive part $A(\lambda)$ an entire function of order $1 / 2$. So with this amplitude there is at most a two-fold ambiguity. This persists as $\lambda$ goes to zero and we do not see how extra solutions could appear out of nowhere. This is an assumption which seems reasonable.

\section{CONCLUDING REMARKS}

We consider that we have solved a longstanding problem. However, purists could object that we rely on a limiting process and that we do not know if a catastrophe could occur in the limit. We do not believe this because the differential cross section remains continuous at the limit. We apply "Goldberger's principle" that "nature cannot be so unkind" [12]. We hope that someone else can make our argument more rigorous. However there is a big problem left which is the existence of solutions. For physical cross sections like $\pi \pi$ scattering, physics tells us that the solution exists, but assuming an arbitrary differential cross section is there at least one acceptable amplitude? It is only under a condition similar to (5), where 0.79 is replaced by 1 [3], that we are certain of the existence of a solution.

\section{ACKNOWLEDGMENTS}

We would like to thank Shirin Davarpanah for her help in preparing the manuscript.

\section{APPENDIX A: BOUND ON THE NUMBER OF AMPLITUDES}

In Ref. [6], we have shown that if the scattering amplitude is a polynomial of degree $L$, and, therefore, the differential cross section a polynomial of degree $2 L$, the scattering amplitude is unique if the cross section is less than 1.38. It is tempting to assume that this result, independent of $L$ is also valid for an infinite number of partial waves. Here, we want to generalize this result and prove that, given the total cross section, one can find a bound on the maximum number of amplitudes compatible with the differential cross section which is independent of $L$ and depends only on the value of the total cross section, while, naively one would expect a bound of $2^{L-1}$. The method is the same as in [6]. It consists in starting with the maximum $\ell$, i.e., $L$, and descending in values of $\ell$. From the Legendre polynomial expansion of the differential cross section:

$$
\sum_{0}^{2 L}(2 \ell+1) C_{\ell} P_{\ell}(\cos \theta),
$$

we see that the value of $\sin \left(\delta_{L}\right)$ is fixed by the knowledge of $C_{2 L}$ (we choose $\delta$ between 0 and $\pi / 2$ ). Then, from $C_{2 L-1}$, we get

$$
\operatorname{Re}\left(f_{L-1} f_{L}^{*}\right)
$$

This defines in the Argand diagram a straight line which intersects the unitarity circle in 2 points (if they do not intersect, the differential cross section is not acceptable). If only one of these 2 points is acceptable (and we shall see that soon) we can continue the procedure down to a value $\ell=M$. Suppose now that at $\ell=M$ we have the 2 solutions, $f_{M}$ and $g_{M}$. Then we have

$$
\operatorname{Re}\left(f_{M}-g_{M}\right) f_{L}^{*}=0 .
$$

So $f_{M}-g_{M}$ is perpendicular to the segment $\left[0, f_{L}\right]$. The point $\left(f_{M}+g_{M}\right) / 2$ is on a line parallel to $\left[0, f_{L}\right]$ going through the center of the unitarity circle. See Fig. 1. It is easy to see that since the segment $\left[f_{M}, g_{M}\right]$ must at least touch the circle we must have

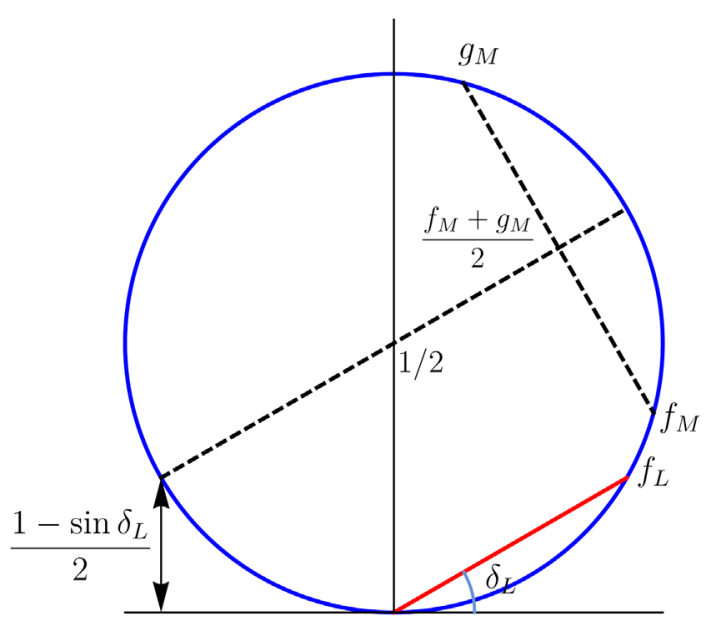

FIG. 1. Notations used in Appendix A. 


$$
\frac{\operatorname{Im} f_{M}+\operatorname{Im} g_{M}}{2}>\frac{1-\sin \delta_{L}}{2} .
$$

So the total cross section, common to both amplitudes, should be larger than

$$
(2 M+1) \frac{1-\sin \delta_{L}}{2}+(2 L+1) \sin ^{2} \delta_{L} .
$$

Minimizing with respect to $\delta_{L}$, and noticing that $M<L$ we get

$$
\sigma_{\text {tot }}>(7 / 8)(M+1 / 2) .
$$

If, on the other hand,

$$
(7 / 8)(M+3 / 2)>\sigma_{\text {tot }}>(7 / 8)(M+1 / 2),
$$

we see that there cannot be any ambiguity for $\ell \geq M+1$, which we anticipated, and we must choose the solution with the smallest imaginary part.

At this stage we can have ambiguities at each step descending to $\ell=1$. As $\ell=0$ is fixed, an upper bound on the number of solutions is

$$
2^{(M-1)}<2^{7 /\left(8 \sigma_{\mathrm{tot}}\right)} .
$$

This bound is independent of $L$. We believe that this also holds for an infinite number of partial waves. What really matters for the rest of this paper is that the number of solutions is finite.

\section{APPENDIX B: PROOF OF THE INEQUALITIES EQ. (13)}

$\left|P_{\ell}(z)\right|>P_{\ell}(|z|$ for $|z|>1$. The Legendre polynomials have zeros between -1 and +1 . Hence, taking for instance $\ell$ even

$$
\left|P_{\ell}(z)\right|=C_{\ell}\left|\prod_{i}\left(z^{2}-x_{i}^{2}\right)\right|>C_{\ell} \prod_{i}\left(|z|^{2}-x_{i}^{2}\right)=P_{\ell}(|z|) .
$$

Now, $P(x)$ for $x$ real larger than 1 can be written as

$$
\begin{aligned}
P_{\ell}(x)= & \frac{1}{\pi} \int_{0}^{\pi / 2}\left[\left(x+\cos \phi \sqrt{x^{2}-1}\right)^{\ell}\right. \\
& \left.+\left(x-\cos \phi \sqrt{x^{2}-1}\right)^{\ell}\right] d \phi>x^{\ell} .
\end{aligned}
$$

This proves the left hand inequality. The second inequality follows from the above integral representation: for $|z| \geq 1$,

$$
\begin{aligned}
\left|P_{\ell}(z)\right| & <\frac{|z|^{\ell}}{\pi} \int_{0}^{\pi}\left(1+\sqrt{1+1 /|z|^{2}}\right)^{\ell} d \phi \\
& <|z|^{\ell}(1+\sqrt{2})^{\ell} .
\end{aligned}
$$

\section{APPENDIX C: ASYMPTOTIC BEHAVIOR OF $\operatorname{Re} r_{\ell}$ FOR LARGE $\ell$}

We have

$$
\operatorname{Re} r_{\ell}=\frac{\lambda}{2} \int_{-1}^{+1} P_{\ell}(x) \exp x d x .
$$

From the definition of Legendre polynomials

$$
P_{\ell}(x)=\frac{1}{2^{\ell} \ell !}\left(\frac{d}{d x}\right)^{\ell}\left(x^{2}-1\right)^{\ell},
$$

we can integrate the right-hand side of $(\mathrm{C} 1) \ell$ times by parts:

$$
\operatorname{Re} r_{\ell}=\frac{\lambda}{2^{\ell} \ell !} \int_{-1}^{+1} \cosh x\left(1-x^{2}\right)^{\ell} d x
$$

This gives for large $\ell$

$$
\begin{aligned}
\operatorname{Re} r_{\ell} & \simeq \frac{\lambda}{2} \frac{1}{2^{\ell} \ell !} \int_{-\infty}^{+\infty} \exp \left[-(\ell-1 / 2) x^{2}\right] d x \\
& =\frac{\lambda}{2} \frac{1}{2^{\ell} \ell !} \sqrt{\frac{\pi}{\ell-1 / 2}} .
\end{aligned}
$$

[1] T. Y. Wu and T. Ohmura, Quantum Theory of Scattering (Englewoods Cliff, NJ, 1962), p. 100.

[2] J. H. Crichton, Phase-shift ambiguities for spin-independent scattering, Nuovo Cimento A 45, 256 (1966); See, also, D. Atkinson, P. W. Johnson, and R. L. Warnock, Determination of the scattering amplitude from the differential crosssection and unitarity, Commun. Math. Phys. 28, 133 (1972); D. Atkinson, G. Mahoux, and F. J. Indurain,
Construction of a unitary analytic scattering amplitude (I). Scalar particles, Nucl. Phys. B54, 263 (1973).

[3] A. Martin, Construction of the scattering amplitude from the differential cross-sections, Nuovo Cimento A 59, 131 (1969).

[4] R. G. Newton, Determination of the amplitude from the differential cross section by unitarity, J. Math. Phys. (N.Y.) 9, 2050 (1968). 
[5] A. D. Gangal and J. Kupsch, Determination of the scattering amplitude, Commun. Math. Phys. 93, 333 (1984).

[6] C. Itzykson and A. Martin, Phase-shift ambiguities for analytic amplitudes, Nuovo Cimento A 17, 245 (1973).

[7] F. A. Berends and S. M. N. Ruijenaars, Examples of phaseshift ambiguities for spinless elastic scattering, Nucl. Phys. 56, 507 (1973).

[8] H. Cornille (private communication).

[9] H. Cornille and J.-M. Drouffe, Phase-shift ambiguities for spinless and $L_{\max } \leq 4$ elastic scattering, Nuovo Cimento A 20, 401 (1974).

[10] R. P. Boas, Entire Functions (Academic Press, New York, 1954).
[11] H. Lehmann, Analytic properties of scattering amplitudes as functions of momentum transfer, Nuovo Cimento 10, 579 (1958); A. Martin, Extension of the axiomatic analyticity domain of scattering amplitudes by unitarity-I, Nuovo Cimento A 42, 930 (1966).

[12] A.S. Wightman, Proceedings of the 14th International Conference on High Energy Physics, Vienna, edited by J. Prentki and J. Steinberger (CERN, Geneva, 1968), p. 434. Goldberger was a great physicist who wrote pionnucleon dispersion relations. His proof was lousy but the result was correct and confirmed by Bogoliubov and his friends. 